\title{
Epidemiologia da violência contra a criança no Estado de Goiás
}

\author{
Epidemiology of violence against children in the State of Goiás \\ Epidemiología de la violencia contra los niños en el Estado de Goiás
}

Kalyne Naves Guimarães Borges ${ }^{1}$, Marília Gabriella Mendes Maranhãoํㅜ, Marília Diniz Inocencio', Marília Miguel de Lucena ${ }^{1}$, Marina Borges de Paula ${ }^{1}$, Rafaela Silva Oliveira ${ }^{1}$, Heloísa Silva Guerra ${ }^{2 *}$.

\section{RESUMO}

Objetivo: Apresentar o perfil epidemiológico dos casos de violência contra crianças menores de 10 anos no Estado de Goiás, no período entre 2009 e 2014. Métodos: Trata-se de estudo descritivo-analítico, cujos dados foram obtidos por meio do preenchimento da ficha de notificação "Violência doméstica, sexual e/ou outras violências", disponível no SINAN. Utilizou-se como amostra crianças menores de 10 anos vítimas de violência no período entre 2009 e 2014 no Estado de Goiás. Foram calculadas as frequências de variáveis segundo as características da ocorrência em relação ao ano, faixa etária e sexo das vítimas, tipo de violência, vínculo do agressor com a vítima, e evolução do caso. Resultados: Obteve-se amostra de 3.258 casos referentes à violência contra a criança no período analisado, na qual houve prevalência da negligência/abandono na faixa etária entre 5 e 9 anos de idade e no sexo feminino, sendo os agressores, principalmente, os pais das crianças. A maioria dos casos de violência infantil evoluiu com alta hospitalar. Conclusão: Estes dados apontam a necessidade de se fortalecer intervenções no sentido de ampliar a rede de proteção social às crianças.

Palavras-chave: Maus-tratos infantis, Violência, Epidemiologia.

\begin{abstract}
Objective: To present the epidemiological profile of cases of violence against children under 10 years old in the State of Goiás, between 2009 and 2014. Methods: This is a descriptive-analytical study, whose data were obtained by completing the data sheet notification "Domestic violence, sexual violence and / or other violence", available at SINAN. We used as a sample children under 10 years of age who were victims of violence in the period between 2009 and 2014 in the State of Goiás. Frequencies of variables were calculated according to the characteristics of the occurrence in relation to the year, age group and sex of the victims, type of violence, the abuser's attachment to the victim, and the evolution of the case. Results: A sample of 3,258 cases of violence against children in the analyzed period was obtained, in which there was a prevalence of neglect / abandonment in the age group between 5 and 9 years of age and in the female sex, being the aggressors mainly the parents of children. The majority of cases of child violence evolved during hospital discharge.
\end{abstract}

Key words: Child Abuse, Violence, Epidemiology.

\section{RESUMEN}

Objetivo: Presentar el perfil epidemiológico de los casos de violencia contra niños menores de 10 años en el estado de Goiás, entre 2009 y 2014. Métodos: Este es un estudio analítico descriptivo, cuyos datos se

\footnotetext{
${ }^{1}$ Acadêmicos de Medicina, Universidade de Rio Verde, Aparecida de Goiânia, Goiás.

${ }_{2}^{2}$ Professora Adjunto I da Faculdade de Medicina da Universidade de Rio Verde, Aparecida de Goiânia, Goiás. *E-mail: heloisasguerra@gmail.com
} 
obtuvieron al completar la hoja de datos. Notificación "Violencia doméstica, violencia sexual y/u otra violencia", disponible en SINAN. Usamos como muestra a niños menores de 10 años que fueron víctimas de violencia en el período de 2009 a 2014 en el estado de Goiás. Las frecuencias de las variables se calcularon según las características de la incidencia en relación con el año, el grupo de edad y el sexo de las víctimas, el tipo de violência, el apego del abusador a la víctima, y la evolución del caso. Resultados: Se obtuvo una muestra de 3.258 casos de violencia contra niños en el período analizado, en los cuales prevaleció el abandono en el grupo de edad entre 5 y 9 años y en el sexo femenino, siendo los agresores principalmente los padres de los niños. La mayoría de los casos de violencia infantil evolucionaron con alta de hospital. Conclusión: estos datos apuntan a la necesidad de fortalecer las intervenciones para ampliar la red de protección social para niños.

Palabras clave: Maltrato Infantil, Violencia, Epidemiología.

\section{INTRODUÇÃO}

A violência contra a criança é um problema de destaque na saúde pública, sendo uma causa recorrente de atendimento ambulatorial pediátrico e hospitalizações, e a principal causa de morte em crianças a partir de 5 anos de idade (MARTINS FF e ROMAGNOLI RC, 2017).

Devido à alta gravidade e morbimortalidade, essa condição recebeu posição de destaque na sociedade e na política, sendo, no fim dos anos 80, concebido o Estatuto da Criança e do Adolescente através da Lei 8.069 tendo por objetivo assegurar às crianças e aos adolescentes, a promoção da saúde e a prevenção de agravos, reputando obrigatória a identificação e a denúncia de violência (BRASIL, 1990).

Os maus tratos às crianças são classificados em quatro categorias pela Organização Mundial de Saúde (OMS): o abuso físico, sexual, emocional/psicológico e negligência, sendo que esta última abrange a saúde, a educação, o desenvolvimento emocional, a nutrição e a habitação ou condições seguras de vida (WHO, 2006).

Segundo o Boletim Epidemiológico do Ministério da Saúde, no ano de 2011, dentre as crianças menores de 10 anos de idade, a negligência foi o tipo de violência mais comum $(43,1 \%)$, seguido da violência física (33,3\%) (BRASIL, 2013).

Na maior parte dos atendimentos, o provável autor da agressão tratava-se de um familiar, sendo a mãe a autora da violência em $36 \%$ dos casos notificados. No mesmo ano, o registro de evolução dos casos notificados mostrou que $89,6 \%$ receberam alta, $61,6 \%$ foram encaminhados para acompanhamento ambulatorial e 198 casos (1,5\%) evoluíram para o óbito (RATES SM, et al., 2015).

A OMS afirma que as consequências da violência infantil vão muito além das disfunções e lesões físicas, envolvendo o desenvolvimento físico e mental das vítimas. Além disso, a exposição à violência durante a infância está associada a fatores e comportamentos de risco durante a vida adulta, como depressão, obesidade, tendência a comportamentos violentos, comportamentos sexuais de alto risco, tabagismo e aumento do consumo de álcool e drogas. Estes fatores de risco podem tornar os indivíduos susceptíveis a enfermidades, como as cardíacas, o câncer, as doenças sexualmente transmissíveis e o suicídio (WHO, 2006).

Assim, é de suma importância que os profissionais da área de saúde conheçam o perfil epidemiológico da violência contra a criança na região em que atuam, visto que tal informação pode ser útil na prevenção da mesma e, dessa forma, auxiliar na diminuição das consequências deste agravo na saúde física e mental das vítimas.

Diante disso, e reconhecendo o papel relevante dos profissionais da área da saúde nesse tipo de contexto, o objetivo desse estudo foi apresentar o perfil epidemiológico dos casos de violência contra crianças menores de 10 anos no Estado de Goiás, no período entre 2009 e 2014. 


\section{MÉTODOS}

Trata-se de um estudo descritivo-analítico, cujos dados foram obtidos por meio do preenchimento da ficha de notificação "Violência doméstica, sexual e/ou outras violências", disponível no Sistema de Informação de Agravos de Notificação (SINAN) do Departamento de Informática do Sistema Único de Saúde (DATASUS).

A população do estudo foi constituída por todos os casos de crianças vítimas de violência com idade até 10 anos, registrados no período de 2009 a 2014. Foram inclusos apenas os casos com ocorrência e notificação no Estado de Goiás, cujas vítimas tinham residência no mesmo.

Foram selecionadas as seguintes variáveis para o estudo: (1) características da ocorrência em relação ao ano; (2) características demográficas das vítimas (faixa etária e sexo); (3) tipo de violência; (4) características do agressor (vínculo com a vítima); e, (5) evolução do caso.

Os dados obtidos no SINAN foram apresentados em tabelas com o número absoluto e percentual das variáveis. A pesquisa utilizou dados secundários disponíveis em sistemas públicos de informações, não sendo necessária a submissão do projeto ao Comitê de Ética em Pesquisa.

\section{RESULTADOS}

Entre o período de 2009 e 2014, foram registrados o total de 3.258 casos referentes à violência contra a criança e foi possível observar oscilações nos números ano a ano (Tabela 1).

Tabela 1 - Distribuição total de número de casos de violência contra a criança entre os anos de 2009 e 2014, segundo dados do SINAN, Goiás, 2018.

\begin{tabular}{lcc}
\hline Ano de incidência & $\mathbf{N}$ & $\%$ \\
\hline 2009 & 99 & 3,0 \\
2010 & 400 & 12,3 \\
2011 & 214 & 6,7 \\
2012 & 680 & 20,9 \\
2013 & 937 & 28,7 \\
2014 & 928 & 28,4 \\
\hline Total & $\mathbf{3 . 2 5 8}$ & $\mathbf{1 0 0}$ \\
\hline Fonte: SINAN/SVS/MS (2018). & &
\end{tabular}

Observa-se que, em relação à faixa etária, o maior número de notificações contempla crianças de faixa etária entre 5 e 9 anos, com $1.396(42,9 \%)$ casos notificados, seguida pela faixa etária entre 1 e 4 anos de idade, com $1.320(40,5 \%)$ notificações. Em relação ao sexo, nota-se que os casos envolvendo meninas totalizaram 1.691 (51,9\%) notificações (Tabela 2).

Tabela 2 - Distribuição do número de casos de violência contra a criança, conforme a faixa etária e sexo, segundo dados do SINAN, Goiás, 2009-2014.

\begin{tabular}{lcccccc}
\hline Faixa etária & \multicolumn{2}{c}{ Masculino } & \multicolumn{2}{c}{ Feminino } & \multicolumn{2}{c}{ Total } \\
\hline \multirow{3}{*}{$>$ ano } & $\mathrm{N}$ & $\%$ & $\mathrm{~N}$ & $\%$ & $\mathrm{~N}$ & $\%$ \\
1-4 anos & 310 & 19,8 & 231 & 13,6 & 541 & 16,6 \\
$5-9$ anos & 585 & 37,3 & 735 & 43,5 & 1320 & 40,5 \\
\hline Total & 671 & 42,9 & 725 & 42,9 & 1396 & 42,9 \\
\hline
\end{tabular}

Fonte: SINAN/SVS/MS (2018). 
A negligência/abandono predomina com 1.476 (32,4\%) das notificações, seguida pela violência física com $1.390(30,3 \%)$ casos. Os dados obtidos sugerem que algumas crianças sofreram mais de uma modalidade de violência. Os índices de violência sexual são expressivos, uma vez que tiveram $999(21,8 \%)$ casos notificados (Tabela 3).

Tabela 3 - Distribuição do número de casos de violência contra a criança, conforme o tipo de violência, segundo dados do SINAN, Goiás, 2009-2014.

\begin{tabular}{lcc}
\hline Tipo de Violência & No & $\%$ \\
\hline Negligência/Abandono & 1.476 & 32,4 \\
Física & 1.390 & 30,3 \\
Sexual & 999 & 21,8 \\
Psicológica/Moral & 595 & 12,9 \\
Tortura & 75 & 1,6 \\
Outros tipos & 48 & 1,0 \\
\hline Total & $\mathbf{4 . 5 8 3}$ & $\mathbf{1 0 0 , 0}$ \\
\hline
\end{tabular}

Fonte: SINAN/SVS/MS (2018).

Dos casos notificados, a mãe surge como a principal agressora em $1.225(38,8 \%)$ casos, seguida do pai com 894 (28,3\%). É importante ressaltar que, na maioria dos casos, os agressores detinham vínculo com as vítimas (Tabela 4).

Tabela 4 - Distribuição do número de casos de violência contra a criança, conforme o vínculo, segundo dados do SINAN, Goiás, 2009-2014.

\begin{tabular}{lcc}
\hline Vínculo & $\mathbf{N} N$ & $\%$ \\
\hline Mãe & 1.225 & 38,8 \\
Pai & 894 & 28,3 \\
Outros vínculos & 412 & 13,0 \\
Desconhecido (a) & 210 & 6,7 \\
Padrasto & 188 & 5,9 \\
Cuidador (a) & 106 & 3,4 \\
Irmão (a) & 104 & 3,3 \\
Madrasta & 20 & 0,6 \\
\hline Total & $\mathbf{3 . 1 5 9}$ & $\mathbf{1 0 0 , 0}$ \\
\hline
\end{tabular}

Fonte: SINAN/SVS/MS (2018).

Quanto aos dados obtidos, a grande maioria evolui com alta hospitalar em $2.573(78,8 \%)$ casos. O número de óbitos notificados não totaliza $1 \%$ dos casos, no entanto, os altos índices de violência contra a criança são alarmantes, haja vista que os números notificados são subestimados (Tabela 5).

Tabela 5 - Distribuição do número de casos de violência contra a criança, conforme a evolução do caso, segundo dados do SINAN, Goiás, 2009-2014.

\begin{tabular}{lll}
\hline Evolução & No & $\%$ \\
\hline Alta hospitalar & 2.573 & 78,8 \\
Ignorado/Branco & 595 & 18,6 \\
Evasão/Fuga & 59 & 1,7 \\
Óbito por violência & 24 & 0,7 \\
Óbito por outra causa & 7 & 0,2 \\
\hline Total & $\mathbf{3 . 2 5 8}$ & $\mathbf{1 0 0 , 0}$ \\
\hline Fota: SINAN/SVS/MS (2018) & &
\end{tabular}

Fonte: SINAN/SVS/MS (2018). 


\section{DISCUSSÃO}

Neste estudo, optou-se por consultar a fonte de informação SINAN, em virtude de ser uma fonte de fácil acesso para a obtenção de dados e informação em saúde, estando disponível a profissionais e pesquisadores.

A violência, principalmente aquela que acontece na fase infantil, é um complexo problema na sociedade com grande impacto na saúde pública, podendo deixar sequelas que se repercutirão na vida adulta das vítimas (NUNES AJ e SALES MCV, 2016). Ela vem mostrando aumentos importantes com o passar dos anos, sendo um fenômeno crescente no mundo, mas cujo conhecimento ainda está em processo de construção em função de sua complexidade (MARTINS CBG, 2010).

A análise dos dados do SINAN (2018) entre os anos de 2009 a 2014 apontaram o ano de 2013 como o de maior número de notificações de violências contra crianças no Estado de Goiás, sendo possível perceber números oscilantes e crescentes de notificações de violência infantil no período. O aumento da quantidade de notificações ocorrido nos últimos anos no Brasil pode gerar influências nestes dados (ALVARES JK, et al., 2015).

Em outra perspectiva, apesar de investimentos para o aumento no número de notificações, a prática notificatória da violência ainda ocorre de forma incipiente no país, não sendo possível conhecer a real magnitude deste problema (MARTINS CB, 2010). Dessa forma, é necessária maior dedicação na prática profissional para o aprimoramento das notificações de modo geral, visando estabelecer dados que, de fato, possam contribuir para a construção de perfis epidemiológicos no país.

Quanto à variável relacionada à faixa etária, este estudo demonstrou que crianças entre 5 e 9 anos representaram o grupo mais susceptível à violência, seguida pela faixa etária entre 1 e 4 anos de idade, o que corrobora com outros achados sobre violência infantil (PFEIFFER L et al., 2011; WAISELFISZ JJ, 2012). Em virtude do grande acesso às crianças, escolas podem ser um valioso instrumento na detecção de casos de violência (APOSTÓLICO MR e NÓBREGA CR, 2012).

Dessa forma, considerando que a data de ingresso das crianças no Ensino Fundamental é a partir dos seis anos de idade, como determina o Parecer o 18 do Ministério da Educação (2005), este pode ter sido um dos fatores que contribuíram para uma maior detecção e notificação dos casos de violência às crianças com idade entre 5 e 9 anos.

Percebeu-se através deste estudo a prevalência de notificações de violência em crianças do sexo feminino em comparação com aquelas do sexo masculino. Este achado corrobora com a literatura que destaca o sexo feminino entre casos de violência contra a criança, principalmente a violência sexual (ALMEIDA LA, et al., 2017; ASSIS SG, et al., 2012; MACHADO JA e SANCHES MA, 2014; PELISOLI C, et al., 2010).

Além disso, esta realidade repercute sobre o fato de que, posteriormente, na vida adulta, existe um domínio da força masculina em opressão à feminina, remetendo ao histórico de uma sociedade patriarcal em que 0 homem, a partir da invenção do arado, tornou-se detentor da propriedade, e à mulher foram impostas a funções cada vez mais afastadas da sociedade (PORTO ML e AMARAL WN, 2014).

Existem muitos tipos de violência, porém, segundo a OMS, a violência contra a criança pode ser classificada em abuso físico, violência sexual, violência emocional ou psicológica, e negligência (NUNES AJ e SALES MCV, 2016).

No Estado de Goiás, os tipos mais prevalentes de violência contra crianças foram: negligência/abandono, violência física e violência sexual. Estudo realizado por Egry EY e Apostólico MR (2015) exemplifica alguns tipos de negligência, tais como a saúde-proteção, proteção, educação, saúde, saúde-educação, estrutural, entre outros, sendo a primeira a mais prevalente.

A negligência/abandono resulta de uma dinâmica estabelecida entre fatores econômicos, sociais, comunitários e pessoais, estando ligada ao aumento da mortalidade infantil, à exposição a outras formas de violência e à restrição na experiência de novas relações sociais (PASIAN MS, et al., 2013). 
Além disso, quanto ao aspecto relacionado ao agressor, os dados deste estudo revelaram que o autor do abuso é quase sempre alguém que detém a responsabilidade pela criança, sendo a mãe a principal agente da violência, seguida pelo pai. Na maioria das vezes, as mães são as principais cuidadoras de seus filhos. Este fato pode explicar o motivo de as mesmas serem indicadas como as principais agentes da violência à criança, já que são as pessoas que mais convivem nesse período, sendo responsável por todos os cuidados que a fase requer (NUNES AJ e SALES MCV, 2016). Conforme ponderado por Machado JC et al. (2014), na maioria das vezes, em caso de separação conjugal, a mãe passa a deter a guarda da criança, sendo, portanto, a principal a autora de violência, principalmente daquela relacionada a castigos físicos e psicológicos (MOURA MS, et al., 2008).

Deve-se ressaltar que o domicílio, ambiente do qual a criança deveria estar segura, pode se tornar um lugar onde elas estão mais vulneráveis à violência, em decorrência de não terem a quem solicitar ajuda (MASCARENHAS DM, et al., 2010). Ainda, é importante destacar que a criança pode se tornar vítima da violência cometida por qualquer membro da família, dependendo do contexto em que vive, até pelo fato de serem dependentes da família em diversos aspectos e, por isso, acabam se tornando indefesas às situações de maus tratos (BRASIL, 2001).

Por último, analisando a evolução gerada pela violência à criança, notou-se que um número considerável de crianças recebeu alta hospitalar. Assim, entende-se que a violência resultou em agravo à saúde com necessidade de atendimento médico-hospitalar. Para Martins CBG e Jorge MHPM (2009), além das consequências à saúde das vítimas - como fraturas, lacerações e traumas, a violência pode gerar distúrbios psicológicos e comportamentais, como o uso de álcool e drogas, delinquência, depressão, fobias, retração nos relacionamentos, suicídio, queda da autoestima, que interferem no crescimento e desenvolvimento e podem fazer das vítimas futuros agressores, evidenciando assim a complexa trama existente na violência (MARTINS CBG, 2010).

Pelo fato de o presente estudo utilizar dados secundários, percebeu-se limitação quanto ao controle de possíveis erros decorrentes de registro, além de possíveis subnotificações. No entanto, acredita-se que, por se tratar de dados nacionais oficiais e de preenchimento obrigatório em todos os serviços de saúde, seus resultados permitiram o alcance dos objetivos propostos, chamando a atenção para o cenário da violência, com intuito de favorecer as intervenções às crianças vítimas de qualquer tipo de violência. Acredita-se que a participação mais efetiva dos profissionais de saúde possa colaborar para a ampliação das iniciativas de atendimento a casos de violência, minimizando esta situação cruel.

De acordo com Egry EY et al. (2017) é visível entre profissionais de saúde, lacunas importantes acerca dos saberes sobre a temática da violência infantil, da legislação vigente, das políticas nacionais e internacionais, da historicidade do desenvolvimento infantil, das mudanças ocorridas na infância, das questões de gênero e das consequências da negligência institucional em relação ao enfrentamento da violência e das possibilidades existentes no enfrentamento do fenômeno. Dessa forma, estudos que se proponham a discutir esta problemática, são válidos.

\section{CONCLUSÃO}

Por meio do presente estudo, foi possível apresentar o perfil epidemiológico dos casos de violência contra crianças menores de 10 anos no Estado de Goiás, no período entre 2009 e 2014. Verifica-se que a notificação da violência contra a criança oscilou ao longo dos anos, sendo prevalente a negligência/abandono em crianças da faixa etária entre 5 e 9 anos de idade e do sexo feminino, tendo como agressores na maioria dos casos os pais das crianças. A maioria das vítimas de violência acaba evoluindo com alta hospitalar, mas ainda é preocupante o número de crianças que evoluem com óbito em decorrência deste complexo fenômeno. Ressalta-se a importância de que os dados coletados pelos serviços de saúde tornem-se cada vez mais completos, a fim de que seus resultados possam subsidiar a criação e melhorias de políticas intervencionistas, além de aprimorar o atendimento dos profissionais da saúde envolvidos no atendimento às crianças vítimas de maus-tratos. Acredita-se no papel fundamental destes profissionais através da participação efetiva no atendimento, integrando o contexto familiar e colaborando para a minimização dos casos de violência infantil. 


\section{REFERÊNCIAS}

1. ALBUQUERQUE LM, et al. Compreendendo a negligência infantil na perspectiva de gênero: estudo em um município brasileiro. Revista da Escola de Enfermagem da USP, 2015; 49(4): 556-563.

2. ALMEIDA LA, et al. A Epidemiologia da violência infantil um estado do nordeste do Brasil: série histórica de 2007 a 2016. Rev. Pre Infec Saúde, 2017; 3(2): 27-33.

3. ALVARES JK, et al. Avaliação da completitude das notificações compulsórias relacionadas ao trabalho registradas por município polo industrial no Brasil, 2007 - 2011. Revista Brasileira de Epidemiologia, 2015;18(1): 123-136.

4. APOSTÓLICO MR, NÓBREGA CR. Características da violência contra a criança em uma capital brasileira. Revista Latino-Americana Enfermagem, 2012: 20(2): [08 telas].

5. APOSTÓLICO MR, et al. Enfrentar a violência infantil na Atenção Básica: como os profissionais percebem? Revista Brasileira Enfermagem, 2017; 70(1): 119-125.

6. ASSIS SG, et al. Notificações de violência doméstica, sexual e outras violências contra crianças no Brasil. Ciência \& Saúde Coletiva, 2012;17(9): 2305-2317.

7. BRASIL. Ministério da Saúde. Secretaria de Vigilância em Saúde. Boletim Epidemiológico. Brasília. Disponível em: http://bvsms.saude.gov.br/bvs/periodicos/boletim_epidemiologico_numero_9_2013.pdf. Acesso em: 08 jun. 2018.

8. BRASIL. Ministério da Saúde. DATASUS. Informações de Saúde. Estatísticas vitais. Sistema de Informação de Agravos de Notificação. Disponível em: http://tabnet.datasus.gov.br/cgi/tabcgi.exe?sinannet/cnv/violebr.def. Acesso em: 28 maio, 2018.

9. BRASIL. Ministério da Educação. Secretaria de Educação Básica. Orientações para a matrícula das crianças de 6 (seis) anos de idade no Ensino Fundamental obrigatório, em atendimento à Lei nำ11.114/ 2005. Diário Oficial da República Federativa do Brasil. Brasília. 2005.

10. BRASIL. Estatuto da Criança e do Adolescente. Câmera dos Deputados, Lei no 8.069, de 13 de julho de 1990. DOU de 16/07/1990. Brasília.

11. BRASIL. Ministério da Saúde. Secretaria de Políticas de Saúde. Violência intrafamiliar: orientações para prática em serviço. Cadernos de Atenção Básica, n. 8. Brasília. 2001.

12. EGRY EY, et al. Compreendendo a negligência infantil na perspectiva de gênero: estudo em um município brasileiro. Rev Esc Enferm USP, 2015; 49(4):556-63.

13. EGRY EY, et al. Enfrentar a violência infantil na Atenção Básica: como os profissionais percebem? Rev Bras Enferm, 2017; 70(1): 113-19.

14. JORGE HP, MARTINS CB. Desfecho dos casos de violência contra crianças e adolescentes no poder judiciário. Acta Paulista de Enfermagem, 2009; 22(6): 800-807.

15. MACHADO JA, SANCHES MA. A Gênese da Violência Infantil. Caderno Teológico da PUCPR, 2014; 2(2): 173-189.

16. MACHADO JC, et al. Violência intrafamiliar e as estratégias de atuação da equipe de Saúde da Família. Saúde Soc., $2014 ; 23$ (3): 828-840.

17. MARTINS CB. Maus tratos contra crianças e adolescentes. Revista Brasileira de Enfermagem, 2010; 63(4): 660665.

18. MARTINS CBG. Maus tratos contra crianças e adolescentes. Rev. bras. enferm., 2010; 63(4): 660-665.

19. MARTINS CBG, JORGE MHPM. Desfecho dos casos de violência contra crianças e adolescentes no poder judiciário. Acta Paulista de Enfermagem, 2009; 22(6): 800-807.

20. MARTINS FF, ROMAGNOLI RC. A violência contra as crianças e adolescentes admitidos no Hospital João XIII: uma análise quantitativa. Revista Interinstitucional de Psicologia, 2017;10(1): 148-161.

21. MASCARENHAS DM, et al. Violência contra a criança: revelando o perfil dos atendimentos em serviços de emergência, Brasil, 2006 e 2007. Caderno Saúde Pública, 2010; 26(2): 347-357.

22. MOURA MS, et al. Detecção de maus-tratos contra a criança: oportunidades perdidas em serviços de emergência na cidade do Rio de Janeiro, Brasil. Caderno Saúde Pública, 2008; 24(12): 2926-2936.

23. NUNES AJ, SALES MCV. Violência contra crianças no cenário brasileiro. Ciênc. saúde coletiva, 2016; 21 (3): 871 880.

24. PASIAN MS, et al. Negligência Infantil: A Modalidade Mais Recorrente de Maus-Tratos. Pensando Famílias, 2013;17(2): 61-70.

25. PELISOLI C, et al. Violência sexual contra crianças e adolescentes: dados de um serviço de referência. Temas em Psicologia, 2010; 18(1): 85-97.

26. PFEIFFER L, et al. Violência contra crianças e adolescentes - proposta de classificação dos níveis de gravidade. Revista Paulista de Pediatria, 2011; 29 (4): 477-482.

27. PORTO ML, AMARAL WN. Violência sexual contra a mulher: Histórico e conduta. Femina, 2014; 42(4): $209-215$.

28. RATES SM, et al. Violência infantil: uma análise das notificações compulsórias, Brasil 2011. Ciência \& Saúde Coletiva, 2015; 20(3): 655-665.

29. WAISELFISZ JJ. Mapa da Violência 2012. Os novos padrões da violência homicida no Brasil. Instituto Sangari, São Paulo. 2012.

30. WHO. World Health Organization and International Society for Prevention of Child Abuse and Neglect. Preventing child maltreatment: a guide to taking action and generating evidence. 2006. 